E14-2005-93

M. A. Kiselev, E. V.Zemlyanaya, V. K. Aswal ${ }^{1}$, R.H.H. Neubert ${ }^{2}$

\title{
WHAT CAN WE LEARN ABOUT THE LIPID VESICLE STRUCTURE FROM THE SMALL-ANGLE NEUTRON SCATTERING EXPERIMENT?
}

Submitted to «European Biophysics Journal»

${ }^{1}$ Solid State Physics Division, Bhabha Atomic Research Centre, Trombay, India

${ }^{2}$ Institute of Pharmaceutical Technology and Biopharmacy, Martin-LutherUniversity, Halle (Saale), Germany 
Киселев М.А. и др.

E14-2005-93

Что можно узнать о структуре липидных везикул из экспериментов

по малоугловому рассеянию нейтронов?

Малоугловое рассеяние нейтронов (МУРН) на популяции однослойных везикул с диаметром 500 и $1000 \AA$ использовалось для определения параметров везикул из димиристоилфосфатидилхолина (ДМФХ) в трех фазах (гелевой, рипл- и жидкой). Параметры везикулярных популяций и внутренняя структура бислоя ДМФХ были определены на основе модели разделенных формфакторов (РФФ). Форма везикул, приготовленных экструзией через поры диаметром 500 А, изменяется от приблизительно сферической в жидкой фазе до эллиптической в рипл- и гелевой фазах. Параметры внутренней структуры бислоя (толщина мембраны, толщина его гидрофобной центральной части, гидратация и поверхностная площадь липидной молекулы) были определены на основе гидрофобногидрофильного (ГГ) приближения для плотности длины рассеяния нейтрона поперек бислоя $\rho(x)$ и на основе приближения $\rho(x)$ ступенчатой функцией (СФ). В рамках ГГ-приближения было показано, что толщина мембраны зависит от ее кривизны в жидкой фазе $\left(T=30^{\circ} \mathrm{C}\right)$. Популяция везикул, приготовленная экструзией через поры диаметром $500 \AA$, имеет средний радиус $275,6 \pm 0,5 \AA$, полидисперсность $27 \%$, толщину мембраны $47,8 \pm 0,2 \AA$ А, толщину гидрофобной центральной части 20,5 0,3 А, поверхностную площадь молекулы ДМФХ $61,0 \pm 0,4 \AA^{2}$ и количество молекул воды на молекулу ДМФХ 11,9 \pm 0,3. Везикулы, приготовленные экструзией через поры диаметром $1000 \AA$ А, имеют полидисперсность $48 \%$ и толщину мембраны 45,6 \pm 0,2 А. СФ-приближение было использовано для описания структуры мембраны ДМФХ в гелевой $\left(T=10^{\circ} \mathrm{C}\right)$ и рипл-фазе $\left(T=20^{\circ} \mathrm{C}\right)$. Везикулы ДМФХ, приготовленные экструзией через $1000 \AA$ поры, имеют толщину

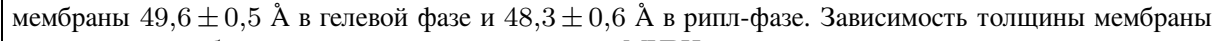
от температуры была определена из эксперимента МУРН.

Работа выполнена в Лаборатории нейтронной физики им. И. М. Франка ОИЯИ.

Препринт Объединенного института ядерных исследований. Дубна, 2005

Kiselev M. A. et al.

E14-2005-93

What Can We Learn about the Lipid Vesicle Structure from the Small-Angle

Neutron Scattering Experiment?

Small-angle neutron scattering (SANS) on the unilamellar vesicle populations (diameter of 500 and $1000 \AA$ A) was used to characterize lipid vesicles from dimyristoylphosphatidylcholine (DMPC) in three phases (gel, ripple, and liquid). Parameters of vesicle populations and internal structure of the DMPC bilayer were characterized on the basis of the Separated Form Factor (SFF) model. Vesicle shape changes from about spherical in liquid phase to elliptical in ripple and gel phases for vesicles prepared via extrusion through pores with the diameter of $500 \AA$ A. Parameters of the internal bilayer structure (membrane thickness, thickness of the hydrophobic core, hydration, and surface area of lipid molecule) were determined on the basis of the Hydrophobic-Hydrophilic $(\mathrm{HH})$ approximation of neutron scattering length density across the bilayer $\rho(x)$ and on the basis of the Step Function (SF) approximation of $\rho(x)$. It was demonstrated in the framework of $\mathrm{HH}$ approximation that DMPC membrane thickness in liquid phase $\left(T=30^{\circ} \mathrm{C}\right)$ depends on the membrane curvature. Vesicle population prepared via extrusion through pores with the diameter of $500 \AA$ is characterized by an average radius of $275.6 \pm 0.5 \AA$, polydispersity of $27 \%$, membrane thickness of $47.8 \pm 0.2 \AA$, thickness of hydrophobic core of $20.5 \pm 0.3 \AA$, surface area per DMPC molecule of $61.0 \pm 0.4 \AA^{2}$, and number of water molecules per DMPC molecule of $11.9 \pm 0.3$. Vesicles prepared via extrusion through pores with the diameter of $1000 \AA$ have a polydispersity of $48 \%$, and a membrane thickness of $45.6 \pm 0.2 \AA$. SF approximation was used to describe the DMPC membrane structure in gel $\left(T=10^{\circ} \mathrm{C}\right)$ and ripple $\left(T=20^{\circ} \mathrm{C}\right)$ phases. DMPC vesicles prepared via extrusion through $1000-\AA$ pores have a membrane thickness of $49.6 \pm 0.5 \AA$ in gel phase and $48.3 \pm 0.6 \AA$ in ripple phase. The dependence of the DMPC membrane thickness on the temperature was restored from the SANS experiment.

The investigation has been performed at the Frank Laboratory of Neutron Physics, JINR.

Preprint of the Joint Institute for Nuclear Research. Dubna, 2005 


\section{INTRODUCTION}

Phospholipids are the main components of cell membranes. Research in the structure of phospholipids is important from a viewpoint of structural biology and biochemistry. Unilamellar vesicles are especially interesting because most biological membranes are unilamellar. Function and properties of integral membrane proteins depend on the lipid bilayer structure. On the other hand, unilamellar vesicles are also used as delivery agents for drugs. The knowledge of its structure in nanoscale region is important for pharmacology (Nagayasu et al. 1999, Cevc et al. 2002).

Dynamic and static light scatterings are commonly used to characterize the form and size of vesicles. However, these methods have the limitation to obtain information about the thickness and the internal structure of the membrane bilayer (Pencer et al. 2001, Jin et al. 1999). The main part of modern knowledge about internal structure of phospholipids in liquid and gel phases was obtained by X-ray diffraction on the giant multilamellar vesicles which have negligibly small membrane curvature (Nagle and Tristram-Nagle 2000). Small-angle neutron scattering (SANS) has been used in many works for the characterization of bilayer structure of the unilamellar vesicles at high excess of water. Membrane thickness can be characterized from experimentally measured radius of gyration of the bilayer by using Guinier approximation (Feigin and Svergun 1987; Knoll et al. 1981; Gordeliy et al. 1993; Balgavy et al. 1998). Calculations of bilayer parameters from the radius of gyration are based on the part of the SANS curve in the interval of scattering vector $q$ from 0.04 to $0.14 \AA^{-1}$. This approach was used for the calculation of bilayer thickness and lipid surface area of the dimyristoylphosphatidylcholine (DMPC) membrane in liquid phase on the base of the Strip Function model of the neutron scattering length density (Kucerka et al. 2004). The electron density profile and the neutron scattering density profile are calculated by Fourier transformation from diffraction peaks intensities in the diffraction experiment on the multilamellar vesicles or oriented dry membranes (Wiener and White 1991; Nagle and Tristram-Nagle 2000; Tristram-Nagle et al. 2002). Accuracy of the structure determination depends on the space resolution of the scattering experiment

$$
\Delta x=\frac{\pi}{q_{m}},
$$

where $q_{m}$ is a maximum value of the measured scattering vector. In the diffraction experiment

$$
\frac{\pi}{q_{m}}=0.5 \frac{d_{u}}{h_{m}}
$$


where $d_{u}$ is repeat membrane thickness and $h_{m}$ is maximum diffraction order (Kiselev et al. 2005). DMPC membrane in access water has $d_{u}=62.7 \AA$ at $T=30^{\circ} \mathrm{C}$ and $\Delta x=4.7 \AA$ for $h_{m}=8$. In the SANS experiment, the coherent scattering intensity of vesicle population can be measured as far as $q_{m} \approx 0.3 \AA^{-1}$. This gives a resolution $\Delta x=10.5 \AA$ of the Fourier transformation, which limits an application of the indirect Fourier transformation for the evaluation of the internal membrane structure from the SANS experiment (Glatter 1977; Glatter 1980). Design of appropriate scattering models and approximations of the scattering length density across the bilayer $\rho(x)$ (based on some preliminary knowledge about bilayer structure) could improve space resolution in the evaluation of the internal membrane structure from the SANS experiment. The model of randomly oriented planar bilayer was applied to characterization of the membrane thickness and the internal membrane structure (Pencer and Hallet 2000; Kucerka et al. 2004). Step function (SF) approximation of $\rho(x)$ was applied to the investigation of oligolamellar vesicles (Schmiedel et al. 2001). This approach could be used to obtain additional information about membrane repeat distance and percentage of nonunilamellar vesicles.

Other important information about vesicle population is average vesicle radius and polydispersity. The Hollow Sphere (HS) model was applied to the calculation of vesicle radius, vesicle polydispersity, membrane thickness and internal membrane structure (Kiselev et al. 2001; Balgavy et al. 2001). The application of the HS model gives possibility to describe the internal membrane structure as two or three regions with constant scattering length density via SF approximation of the $\rho(x)$. The HS model has two principal drawbacks: a) it can be used only for spherical shape of vesicles, b) $\rho(x)$ can be described only as step function, whereas neutron diffraction experiments demonstrate that $\rho(x)$ has a more complex and smooth shape (Wiener and White 1991; Gordeliy and Kiselev 1995).

A model of Separated Form Factors (SFF) allows one to simulate $\rho(x)$ using almost any functions, which significantly expands the possibilities of studying the internal membrane structure (Kiselev et al. 2002). Parameters calculated within the SFF model were the average vesicle radius $\langle R\rangle$, the relative standard deviation of radius (polydispersity) $\sigma$, the membrane thickness $d$, the thickness of hydrophobic part of bilayer $D$, the thickness of hydrophilic part $(D-d) / 2$, and the number of water molecules within a bilayer per DMPC molecule $n_{w}$ (Zemlyanaya and Kiselev 2002; Zemlyanaya et al. 2005). The parameters of DMPC vesicle shape at $T=30^{\circ} \mathrm{C}$ do not depend sufficiently on the different functions used for modeling $\rho(x):\langle R\rangle=275 \pm 0.4 \AA$ and $\sigma=27 \%$ (Zemlyanaya and Kiselev 2002; Zemlyanaya et al. 2005); $\langle R\rangle=272 \pm 0.4 \AA$ and $\sigma=27 \%$ (Kiselev et al. 2004). The values of $d, D$, and $n_{w}$ are however sensitive to the type of function $\rho(x)$ (Kiselev et al. 2004, Kucerka et al. 2004). 
$\mathrm{D}_{2} \mathrm{O}$ penetration inside the bilayer makes part of the bilayer invisible relative to the bulk $\mathrm{D}_{2} \mathrm{O}$ in the case of homogeneous approximation, $\rho(x)=$ const (Kiselev et al. 2004). Therefore, the case of $\rho(x)=$ const gives underestimated value of $d=36.70 \pm 0.02 \AA$ for DMPC vesicles at $T=30^{\circ} \mathrm{C}$ (Zemlyanaya et al. 2005). Calculations based on the SF approximation of $\rho(x)$ give more reasonable value $d=46.4 \pm 0.03 \AA$ and an additional introduction of the linear water distribution function in hydrophilic region of DMPC increases the membrane thickness $d=47.4 \pm 0.04 \AA$ (Zemlyanaya et al. 2005). Generation of $\rho(x)$ based on the Gaussian functions gives $d=50.6 \pm 0.8 \AA$ (Kiselev et al. 2004).

Water distribution function across the bilayer has a sigmoidal form (Armen et al. 1998, Kiselev et al. 2004). In liquid phase, the contribution from the water distribution function to the integrated scattering length density of DMPC is sufficiently larger than the contribution from the polar head group (Kiselev et al. 2004). Competition between contributions of $\mathrm{D}_{2} \mathrm{O}$ and polar head groups to the integrated scattering length density allows one to simplify the approximation of $\rho(x)$ and decrease the number of fit parameters.

In present work, the SFF model is used to analyze the structure of the polydispersed population of DMPC vesicles in gel, liquid, and crystalline phases as studied by SANS. Two types of $\rho(x)$ functions are used for the evaluation of bilayer parameters, which depend on the bilayer hydration. Parameters of the vesicle population $(\langle R\rangle, \sigma$ for spherical shape or $\langle a\rangle, \varepsilon, \sigma$ for elliptical shape) and parameters of bilayer $\left(d, D, n_{W}\right)$ are calculated for the case of vesicles prepared via extrusion through 500- and 1000- $\AA$ pores. The presented methods show what can we learn about vesicle structure from the SANS experiment for other type of lipid vesicles and vesicular-based drug delivery systems.

\section{MATERIALS AND METHODS}

Sample Preparation. DMPC was a gift from Lipoid (Moscow, Russia). $\mathrm{D}_{2} \mathrm{O}$ (99.9\% deuteration) was from Isotop (St. Petersburg, Russia). Samples for measurements were prepared by conventional extrusion technique. Heavy water and DMPC were mixed in a plastic tube and the tube was sealed. The DMPC concentration in the sample was $15 \mathrm{mM}$ (about $1 \mathrm{wt} \%$ ). The tube content was heated to a temperature above the main phase transition temperature and then cooled down to about $-20^{\circ} \mathrm{C}$. The cooling-heating cycle accompanied by sample shaking was repeated four times. From the dispersion of multilamellar vesicles thus obtained, extruded unilamellar vesicles were prepared in a singlestep procedure according to (MacDonald et al. 1991) using the LiposoFast Basic extruder (Avestin, Ottawa, Canada). The multilamellar vesicle populations were prepared by extrusion through one polycarbonate filter (Nucleopore, Plesanton, USA) with pores of diameter $500 \AA$ (namely 500-A extruded vesicles) or $1000 \AA$ 
(namely 1000-Å extruded vesicles), mounted in the extruder fitted with two gastight Hamilton syringes (Hamilton, Reno, USA). The sample was subjected to 25 passes through the filter at a temperature above the main phase transition temperature of the lipid dispersion. An odd number of passes were performed to avoid contamination of the sample by large and multilamellar vesicles, which might not have passed through the filter. The sample was filled into a quartz cuvette (Hellma, Müllheim, Germany) with a 2-mm sample thickness.

SANS Measurements. SANS spectra from unilamellar vesicles were collected as function of temperature in the range of $10-60^{\circ} \mathrm{C}$ at YuMO small-angle time-of-flight spectrometer of Frank Laboratory of Neutron Physics (JINR, Dubna, Russia) in the range of scattering vector $q$ from 0.02 to $0.15 \AA^{-1}$. (Ostanevich 1988). SANS spectra from unilamellar vesicles were collected at $T=10,20$, and $30^{\circ} \mathrm{C}$ at SANS-1 spectrometer of the Swiss Spallation Neutron Source at the Paul Scherrer Institute (PSI), Switzerland. Three sample-to-detector distances of 2,6 , and $20 \mathrm{~m}$ were used to obtain the SANS data over a wide $q$ range from 0.0033 to $0.56 \AA^{-1}$. Neutron wavelength was $4.7 \AA$.

Evaluation of Vesicle Parameters from SANS Curve. The simplest method of membrane thickness characterization is Guinier approximation of scattering curve (Feigin and Svergun 1987; Knoll et al. 1981; Gordeliy et al. 1993; Balgavy et al. 1998). The macroscopic cross section of vesicle population with $R>d$ can be presented as (Gutberlet et al. 2000)

$$
\frac{d \Sigma}{d \Omega}(q)=n \cdot \frac{d \Sigma}{d \Omega}(0) \cdot q^{-2} \cdot \exp \left(-R_{t}^{2} \cdot q^{2}\right),
$$

where $n$ is a number of vesicles per unit volume, and

$$
R_{t}^{2}=\frac{d_{G}^{2}}{12}
$$

Radius of gyration $R_{t}$ is determined from Guinier plot $\left(\frac{d \Sigma}{d \Omega} \cdot(q) \cdot q^{2}\right.$ vs. $\left.q^{2}\right)$, and membrane thickness parameter $d_{G}$ is calculated from Eq. (4) (Gordeliy et al. 1993; Balgavy et al. 1998). The vesicle radius and polydispersity can not be determined in the Guinier approximation. Equation (4) is valid for the case of large value of contrast, when scattering length density of $\mathrm{D}_{2} \mathrm{O}$ is larger than the average scattering length density of the bilayer. It is true for the case of «dry» bilayer, when penetration of $\mathrm{D}_{2} \mathrm{O}$ molecules inside the bilayer is negligibly small (Ibel and Stuhrmann 1975, Gordeliy et al. 1993). For DMPC vesicles in $\mathrm{D}_{2} \mathrm{O}$, the penetration of water molecules in the hydrophilic part of the bilayer influences the scattering length density distribution (Kiselev et al. 2004, Zemlyanaya et al. 2005). It is the reason why $d_{G}$ is smaller relative to the real membrane thickness $d$ (Balgavy et al. 1998, Kiselev et al. 2001). Values of $d_{G}$ and $d$ are 
different by some constant $\Delta d_{H}$ depending on a membrane type and hydration

$$
d=d_{G}+\Delta d_{H} .
$$

The model of Separated Form Factors (SFF) was proposed for the evaluation of the vesicle radius, the polydispersity and the internal membrane structure (Kiselev et al. 2002). The coherent macroscopic cross section of the monodispersed population of vesicles is defined by the formula

$$
\frac{d \Sigma}{d \Omega}_{\text {mon }}(q)=n \cdot A^{2}(q) \cdot S(q)+I B,
$$

where $n$ is the number of vesicles per unit volume, $A(q)$ is the scattering amplitude of a vesicle, IB is the incoherent background, and $S(q)$ is the vesicle structure factor (Feigin and Svergun 1987, Kiselev et al. 2003a). For spherical unilamellar vesicle with radius $R$ (Kiselev et al. 2002, Zemlyanaya et al. 2005),

$$
A(q)=4 \pi \cdot \int_{-d / 2}^{d / 2} \rho_{c}(x) \cdot \frac{\sin [(R+x) \cdot q]}{(R+x) \cdot q} \cdot(R+x)^{2} \cdot d x,
$$

where $\rho_{c}(x)=\rho(x)-\rho_{\mathrm{D}_{2} \mathrm{O}}$ is a contrast, the difference between scattering length densities of the bilayer $\rho(x)$ and the heavy water $\rho_{\mathrm{D}_{2} \mathrm{O}}$. Integration of Eq. (7) gives exact expression for scattering amplitude of vesicle with separated parameters $R, d, \rho(x)$ :

$$
\begin{aligned}
A_{\mathrm{ves}}(q)=4 \pi \cdot \frac{R^{2}}{q R} \cdot & \sin (q R) \cdot \int_{-d / 2}^{d / 2} \rho_{c}(x) \cdot \cos (q x) \cdot d x+ \\
& +4 \pi \cdot \frac{R}{q R} \cdot \cos (q R) \cdot \int_{-d / 2}^{d / 2} \rho_{c}(x) \cdot x \cdot \sin (q x) \cdot d x .
\end{aligned}
$$

In the case of $R \gg d / 2, R+x \approx R$, one can neglect the second term with respect to the first term in Eq. (8) (Kiselev et al. 2002, Zemlyanaya et al. 2005). The first term in Eq. (8) presents the SFF model of the SANS scattering from vesicle. The macroscopic cross section of monodispersed population of vesicles in the framework of the SFF model is written as

$$
\frac{d \Sigma}{d \Omega \text { mon }}(q)=n \cdot F_{s}(q, R) \cdot F_{b}(q, d) \cdot S(q)+I B,
$$

where $F_{s}(q, R)$ is a form factor of the infinitely thin sphere with radius $R$

$$
F_{s}(q, R)=\left(4 \pi \cdot \frac{R^{2}}{q R} \cdot \sin (q R)\right)^{2}
$$


$F_{b}(q, d)$ is a form factor of the symmetric lipid bilayer.

$$
F_{b}(q, d)=\left(\int_{-d / 2}^{d / 2} \rho_{c}(x) \cdot \cos (q x) \cdot d x\right)^{2} .
$$

The SFF model allows characterization of the deformations of the vesicle shape from spherical to elliptical. This case, instead of $F_{s}(q, R)$, the form factor of infinitely thin ellipse $F_{E}(q, a)$ is written as

$$
F_{E}(q, a)=\int_{0}^{1} A_{E}^{2}\left(q \cdot a \cdot \sqrt{1+x^{2} \cdot\left(\varepsilon^{2}-1\right)}\right) d x,
$$

where $\varepsilon$ is an ellipse eccentricity, $a$ is a minor semiaxis, and the function $A_{E}(z)=$ $4 \pi \cdot \varepsilon \cdot a^{2} \cdot \sin (z) / z$.

The polydispersity of vesicle population is described by nonsymmetrical Schulz distribution (Hallet et al. 1991, Schmiedel et al. 2001)

$$
G(R,\langle R\rangle)=\frac{R^{m}}{m !} \cdot\left(\frac{m+1}{\langle R\rangle}\right)^{m+1} \cdot \exp \left[-\frac{(m+1) \cdot R}{\langle R\rangle}\right]
$$

where $\langle R\rangle$ is an average vesicle radius and $m$ is a coefficient of polydispersity. Relative standard deviation of the vesicle radius is given by

$$
\sigma=\sqrt{\frac{1}{(m+1)}}
$$

Thus, the coherent macroscopic cross section of polydispersed vesicle population $I_{\text {theor }}(q,\langle R\rangle, d)$ is calculated as

$$
I_{\text {theor }}(q,\langle R\rangle, d)=\frac{\int_{R \min }^{R \max } \frac{d \Sigma}{d \Omega}{ }_{\text {mon }}^{R}(q, R, d) \cdot G(R,\langle R\rangle) \cdot d R}{\int_{R \text { min }}^{R \max } G(R,\langle R\rangle) \cdot d R},
$$

where $R_{\min }$ and $R_{\max }$ depend on the diameter of a polycarbonate filter.

The experimentally measured macroscopic cross section $I_{\exp }(q)$ is not completely equal to theoretically calculated value of the coherent macroscopic cross section $I_{\text {theor }}(q,\langle R\rangle, d)$ due to the incoherent scattering background IB from a sample and due to the spectrometer resolution distortions. The experimentally 
measured macroscopic cross section $(d \Sigma / d \Omega) \cdot(q)$ is approximated with good accuracy as

$$
\frac{d \Sigma}{d \Omega}(q)=I_{\text {theor }}(q,\langle R\rangle, d)+\frac{1}{2} \cdot \Delta^{2} \cdot \frac{d^{2} I_{\text {theor }}(q,\langle R\rangle, d)}{d q^{2}}+I B
$$

for the case of $\Delta / q \leqslant 0.2$, where $\Delta^{2}$ is a second moment of a spectrometer resolution function (Ostanevich 1988).

Equation (16) was used to fit the SANS data by the $\chi^{2}$ minimizing package DFUMIL from the JINRLIB library (Silin 1967). The codes for fitting were developed in (Kiselev et al. 2004, Zemlyanaya et al. 2005). The fitting parameters are the value of incoherent background IB, number of vesicles per unit volume $n$, average vesicle radius $\langle R\rangle$ for spherical vesicles or average value of minor semiaxis $a$ along with eccentricity $\varepsilon$ for elliptical vesicles, coefficient of polydispersity $m$, thickness of the lipid bilayer $d$, and parameters of function $\rho(x)$.

\section{RESULTS AND DISCUSSION}

Dependence of the Membrane Thickness on Temperature, Guinier Approximation. Figure 1 presents the dependence of the DMPC membrane thickness parameter $d_{G}$ on temperature. DMPC vesicles were prepared by extrusion through pores with diameter of $1000 \AA$. SANS spectra were collected at YuMO spectrometer. Values of $d_{G}$ were evaluated from the Guinier plot via Eqs. (3) and (4). Membrane thickness parameter $d_{G}$ is found to be $44.2 \pm 0.8 \AA$ at $T=10^{\circ} \mathrm{C}$ and $43.4 \pm 0.8 \AA$ at $T=$ $20^{\circ} \mathrm{C}$. The value of $d_{G}$ of $38.8 \pm 0.8 \AA$ at $T=30^{\circ} \mathrm{C}$ was calculated via data extrapolation in Fig. 1. These values of $d_{G}$ give underestimated values of the membrane thickness $d$ relative to the data from X-ray diffraction experiment which are $48.2 \AA$ at $T=10^{\circ} \mathrm{C}$ and $44.2 \AA$ at $T=30^{\circ} \mathrm{C}$ (Nagle and Tristram-Nagle 2000, Tristram-Nagle et al. 2002). The values of $\Delta d_{H}$ in Eq. (5) are equal to $4 \AA$ in gel and

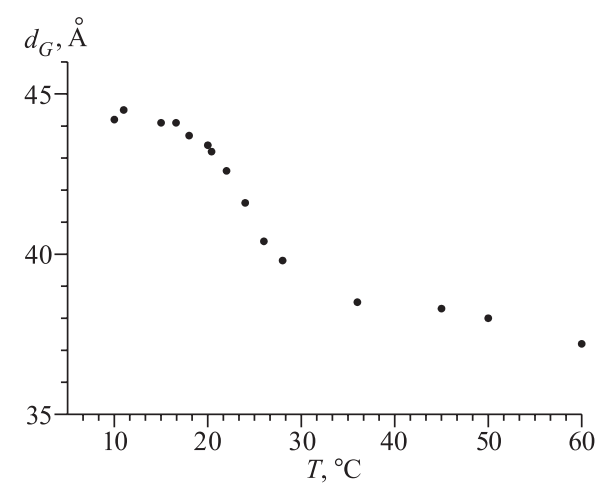

Fig. 1. Dependence of the DMPC membrane thickness parameter $d_{G}$ on temperature for the 1000- $\AA$ extruded vesicles. Values of $d_{G}$ were evaluated from SANS curves in the Guinier approximation $5.4 \AA$ in liquid phases of DMPC, which reflects a larger DMPC hydration in liquid phase. Guinier approximation describes well the relative changes in the membrane thickness under temperature alteration. 
Figure 1 demonstrates the decrease of the membrane thickness at the main phase transition temperature of $23^{\circ} \mathrm{C}$. The membrane thickness decreases by the value of $5.4 \pm 1.6 \AA$ on heating from 10 to $30^{\circ} \mathrm{C}$ and is in agreement with $4-\AA$ decrease obtained from the X-ray experiment.

DMPC Vesicles in the Liquid Crystalline Phase, the HH Approximation of $\rho(x)$. The hydrophobic-hydrophilic (HH) approximation of the internal bilayer structure is used as $\rho(x)$ function in the liquid phase of DMPC (Schmiedel et al. 2004). The HH approximation is based on the fact that the scattering length density of $\mathrm{D}_{2} \mathrm{O}$ molecules in the hydrophilic region of bilayer is sufficiently larger than the scattering length density of polar head groups in liquid phase of DMPC (Kiselev et al. 2004). The HH approximation of $\rho(x)$ is presented in Fig. 2. Lipid bilayer consists of two parts: hydrophilic and hydrophobic. Linear approximation is used for the water distribution function in the hydrophilic region, where $\rho_{\mathrm{D}_{2} \mathrm{O}}=6.4 \cdot 10^{10} \mathrm{~cm}^{-2}$ and $\rho_{\mathrm{CH}}=-0.36 \cdot 10^{10} \mathrm{~cm}^{-2}$ are fixed parameters (Schmiedel et al. 2001, Zemlyanaya et al. 2005). The parameters of the DMPC bilayer determined from the SANS curve are the membrane thickness $d$ and the thickness of the hydrophobic part $D$.

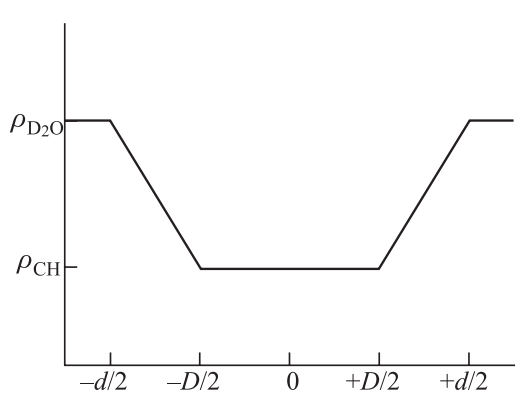

Fig. 2. Hydrophobic-Hydrophilic (HH) approximation of the scattering length density across the lipid bilayer $\rho(x)$; is the membrane thickness, and $D$ is the thickness of the hydrophobic part of the membrane

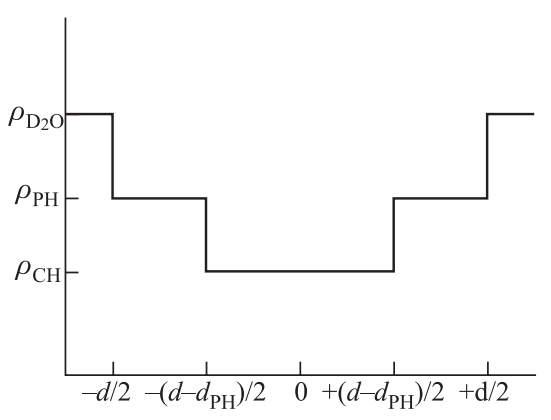

Fig. 3. Step function (SF) approximation of the scattering length density across the lipid bilayer $\rho(x)$; $d$ is the membrane thickness, and $d_{\mathrm{PH}}$ is the thickness of the polar head group, $\rho_{\mathrm{PH}}$ is scattering length density in the region of the polar head group

The number of water molecules $N_{W}$ and the surface area per DMPC molecule $A$ are determined from the system

$$
\left\{\begin{array}{l}
A \cdot \frac{d}{2}=V_{\mathrm{DMPC}}+N_{W} \cdot V_{\mathrm{D}_{2} \mathrm{O}} \\
A \cdot\left(\rho_{\mathrm{CH}} \cdot \frac{D}{2}+\frac{\rho_{\mathrm{D}_{2} \mathrm{O}}-\rho_{\mathrm{CH}}}{2} \cdot \frac{d-D}{2}\right)=l_{\mathrm{DMPC}}+N_{W} \cdot l_{\mathrm{D}_{2} \mathrm{O}},
\end{array}\right.
$$


where $V_{\mathrm{DMPC}}=1101 \AA^{3}$ and $V_{\mathrm{D}_{2} \mathrm{O}}=30 \AA^{3}$ are molecular volumes of DMPC and $\mathrm{D}_{2} \mathrm{O}, l_{\mathrm{DMPC}}=3.07 \cdot 10^{-12} \mathrm{~cm}$ and $l_{\mathrm{D}_{2} \mathrm{O}}=1.92 \cdot 10^{-12} \mathrm{~cm}$ are scattering lengths of the DMPC and $\mathrm{D}_{2} \mathrm{O}$ molecules, respectively. Let us denote $l^{\prime}=$ $l_{\mathrm{DMPC}} / l_{\mathrm{D}_{2} \mathrm{O}}$ and $V^{\prime}=V_{\mathrm{DMPC}} / V_{\mathrm{D}_{2} \mathrm{O}}$. One can define the average scattering length density of hydrated DMPC as

$$
\bar{\rho}=\frac{D}{d} \cdot \rho_{\mathrm{CH}}+\frac{d-D}{d} \cdot \frac{\rho_{\mathrm{D}_{2} \mathrm{O}}-\rho_{\mathrm{CH}}}{2} .
$$

The solution of Eqs. (17) and (18) is

$$
N_{W}=\frac{\bar{\rho} \cdot V^{\prime}-\rho_{\mathrm{D}_{2} \mathrm{O}} \cdot l^{\prime}}{\rho_{\mathrm{D}_{2} \mathrm{O}}-\bar{\rho}}, \quad A=2 \cdot \frac{V_{\mathrm{DMPC}}+N_{W} \cdot V_{\mathrm{D}_{2} \mathrm{O}}}{d} .
$$

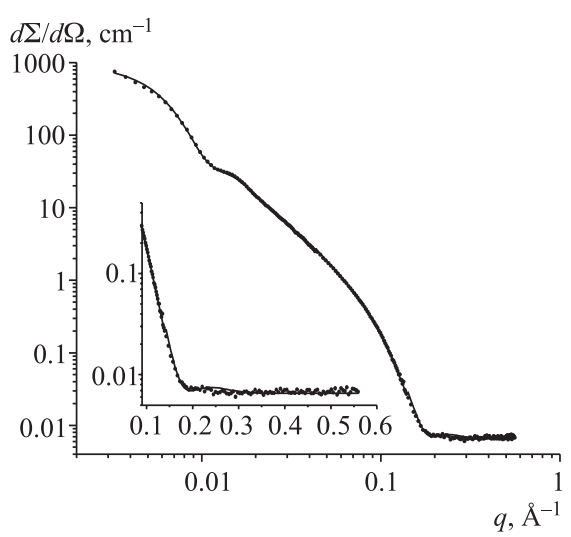

Fig. 4. Experimental macroscopic cross section of the unilamellar vesicle population at $T=30^{\circ} \mathrm{C}$ (dots) for vesicles extruded through pores of $500-\AA$ diameter and fitting curve (solid line). The inset shows the magnified curve for large $q$

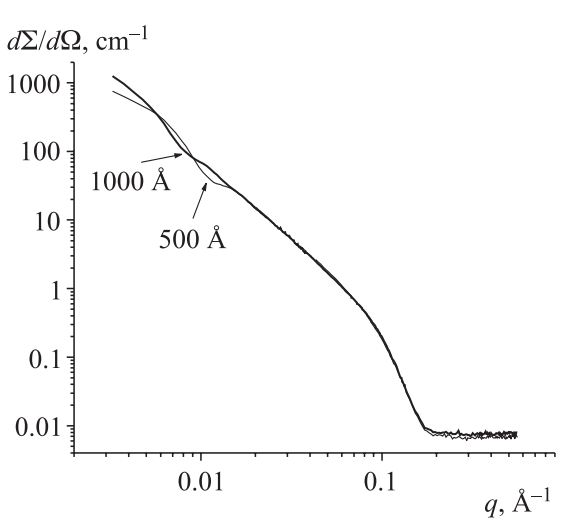

Fig. 5. Experimental macroscopic cross sections of the unilamellar vesicle populations at $T=30^{\circ} \mathrm{C}$ for vesicles prepared by extrusion through pores of 500- and 1000- $\AA$ diameters

Figure 4 shows the experimentally measured and fitted SANS curves for the DMPC unilamellar vesicles at $T=30^{\circ} \mathrm{C}$. Unilamellar vesicles were prepared by extrusion through pores with diameter of $500 \AA$ A . Experimentally measured macroscopic cross section was calculated by two different methods: by application of the exact expression for scattering amplitude using Eq. (8) and by application of the SFF model using Eq. (9). This allows one to check the validity of the SFF model and estimate the contribution of the second term in Eq. (8). The obtained parameters are given in Table 1. Parameters of vesicle populations and internal membrane structure evaluated by the exact expression and by the SFF model for 
Table 1. Results for DMPC vesicles in liquid phase $\left(T=30^{\circ} \mathrm{C}\right)$ based on the $\mathbf{H H}$ approximation of $\rho(x) . D_{F}$ is diameter of the pores used at extrusion, $\langle R\rangle$ is average vesicle radius, $\sigma$ is vesicle polydispersity, $d$ is membrane thickness, $D$ is thickness of the hydrophobic core, $N_{W}$ and $A$ are number of water molecules and surface area per DMPC molecule, IB is value of incoherent background

\begin{tabular}{|c|c|c|c|c|c|c|c|c|}
\hline Model & $D_{F}, \AA$ & $\langle R\rangle, \AA$ & $\sigma, \%$ & $d, \AA$ & $D, \AA$ & $N_{W}$ & $A, \AA^{2}$ & $\mathrm{IB}, \mathrm{cm}^{-1}$ \\
\hline SFF & 500 & $275.6 \pm 0.5$ & 27 & $47.8 \pm 0.2$ & $20.5 \pm 0.3$ & $11.9 \pm 0.3$ & $61.0 \pm 0.4$ & 0.007 \\
Exact & 500 & $275.7 \pm 0.4$ & 27 & $47.8 \pm 0.2$ & $20.5 \pm 0.3$ & $11.9 \pm 0.3$ & $61.0 \pm 0.4$ & 0.007 \\
SFF & 1000 & $314.6 \pm 0.7 *$ & 48 & $45.5 \pm 0.6$ & $20.8 \pm 0.4$ & $10.8 \pm 0.4$ & $62.6 \pm 1.0$ & 0.007 \\
\hline
\end{tabular}

scattering amplitude coincide exactly. Consequently, the second term in Eq. (8) is negligibly small for vesicles with $\langle R\rangle \geqslant 250 \AA$.

For $500-\AA$ extruded vesicles, the obtained value of $\langle R\rangle=275.6 \pm 0.5 \AA$ is in agreement with radius of the pores $(250 \AA)$ used during vesicle extrusion. Membrane thickness $d$ evaluated on the basis of the HH approximation of $\rho(x)$ is equal to $47.8 \pm 0.2 \AA$ and thickness of the hydrophobic region $D$ is equal to $20.5 \pm 0.3 \AA$ A. The thickness of membrane hydrophilic part $D_{H}=(d-D) / 2=$ $13.7 \pm 0.5 \AA$ is larger than the thickness of polar head groups $D_{\mathrm{PH}}=9 \AA$ evaluated from X-ray diffraction (Nagle and Tristram-Nagle 2000) or $D_{\mathrm{PH}}=$ $8.1 \pm 1.7 \AA$ A evaluated from SANS (Kiselev et al. 2004). This result shows that water molecules penetrate the hydrocarbon chain region $4.7 \AA$. The value of membrane thickness $d=47.8 \pm 0.2 \AA$ is $3.6 \AA$ larger than $d=44.2 \AA$ evaluated from X-ray diffraction experiment on the giant multilamellar vesicles (Nagle and Tristram-Nagle 2000). The number of water molecules $N_{W}=11.9 \pm 0.3$ and surface area $A=61.0 \pm 0.4 \AA^{2}$ of the DMPC molecule are different from values $N_{W}=7.2$ and $A=59.6 \AA^{2}$ for the giant multilamellar vesicles (Nagle and Tristram-Nagle 2000). Hydration and membrane thickness of the curved DMPC bilayer are larger relative to the flat bilayer.

Table 2. Results for 500-Å extruded DMPC vesicles in liquid phase $\left(T=30^{\circ} \mathrm{C}\right)$ based on the HH approximation of $\rho(x)$ and elliptical deformations. $\langle a\rangle$ is average value of minor semiaxis, $\varepsilon$ is eccentricity, $\sigma$ is vesicle polydispersity, $d$ is membrane thickness, $D$ is thickness of the hydrophobic core, $N_{W}$ and $A$ are number of water molecules and surface area per DMPC molecule, IB is value of incoherent background. The SFF model

\begin{tabular}{|c|c|c|c|c|c|c|c|}
\hline$\langle a\rangle, \AA$ & $\varepsilon$ & $\sigma, \%$ & $d, \AA$ & $D, \AA$ & $N_{W}$ & $A, \AA^{2}$ & $\mathrm{IB}, \mathrm{cm}^{-1}$ \\
\hline $266 \pm 2$ & $1.11 \pm 0.02$ & 26 & $48.9 \pm 0.2$ & $19.9 \pm 0.4$ & $12.8 \pm 0.3$ & $60.7 \pm 0.5$ & 0.007 \\
\hline
\end{tabular}


The form of vesicles in the liquid phase cannot be ideally spherical. Probably, the vesicle shape fluctuates near the spherical form. These fluctuations come from the unilamellar vesicle deformation and orientation in the strong magnetic field. Unilamellar vesicles deformation in the strong magnetic field of $4 \mathrm{~T}$ has been detected via SANS (Kiselev, unpublished). The elliptical form of the vesicle shape can be taken into account on the basis of Eq. (12). Table 2 presents the results for the case of 500-A extruded vesicles on the assumption of their elliptical form. The value of eccentricity is 1.1 , which demonstrates that vesicle shape is near the sphere. Nevertheless, the membrane thickness has a value of $48.9 \pm 0.2 \AA$ at $\varepsilon=1.1$ in comparison with $d=47.8 \pm 0.2 \AA$ for $\varepsilon=0$.

Important membrane parameter is the thickness of the polar head group $d_{\mathrm{PH}}$. The information about $d_{\mathrm{PH}}$ is hard to be obtained in the framework of the $\mathrm{HH}$ approximation. The large value of scattering length density of $\mathrm{D}_{2} \mathrm{O}$ molecules screens the contribution from scattering length density of polar head groups (Kiselev et al. 2004). A fitting procedure with $\rho(x)$ as sum of linear and step functions gives $d=47.4 \pm 0.2 \AA$ and $D=17.3 \pm 0.5 \AA$ (Zemlyanaya et al. 2005). The number of fitting parameters could be reduced by constrained value of $d_{\mathrm{PH}}=9 \AA$ (Kucerka et al. 2004). This approach gives $d=44.5 \pm 0.3 \AA$, $N_{W}=6.8 \pm 0.3$, and $A=58.9 \pm 0.8 \AA^{2}$ at fitting of SANS curve in the range of $q$ from 0.04 to $0.14 \AA^{-1}$. Accuracy of the membrane thickness evaluation depends on the possibility to collect SANS curve in the region of $q$ corresponding to the bilayer form factor $F_{b}(q, d)$. Roughly, the first minimum of $F_{b}(q, d)$ corresponds to $q_{m}=2 \pi / d$, and for $d=45 \AA q_{m}=0.14 \AA^{-1}$. The evaluation of the bilayer parameters could be improved by the measurements of SANS curve with maximum value of scattering vector $q_{\max }$ about $0.3 \AA^{-1}$ as seen from the insert in Fig. 3. Data acquisition in the $q$ range from 0.30 to $0.56 \AA^{-1}$ is necessary for the evaluation of the incoherent background IB. Determination of IB as fit parameter improves accuracy of the membrane thickness evaluation from SANS curve (Schmiedel et al. 2001). The enlargement of $q_{\max }$ from 0.2 to $0.56 \AA^{-1}$ changes the value of $d$ from $42.5 \pm 0.3$ to $47.4 \pm 0.2 \AA$ and the value of $D$ from $11.0 \pm 0.9$ to $17.3 \pm 0.5 \AA$ A (Zemlyanaya et al. 2005).

Membrane thickness $d=45.5 \pm 0.6 \AA$ is found for the unilamellar vesicles prepared by extrusion through pores with diameter of $1000 \AA$. This value of membrane thickness is in better agreement with $d=44.2 \AA$ obtained for giant multilamellar vesicles. Vesicles prepared via extrusion through pores 500 and $1000 \AA$ are different in the membrane curvature and the polydispersity. The polydispersity (relative standard deviation of radius) increases from 27 to $47 \%$ with increasing vesicle radius as seen from Fig. 4. The evaluated value of the average vesicle radius $\langle R\rangle=314.6 \pm 0.7$ is sufficiently smaller than the radius of the polycarbonate pores $(500 \AA)$ and should be considered as an artifact. Dynamic light scattering (DLS) and freeze-fracture electron microscopy results show that average vesicle radius after extrusion is about the radius of the pores 
(MacDonald et al. 1991, Hallet et al. 1991, Patty and Frisken, 2003). The underestimation of the vesicle radius for 1000 - $\AA$ extruded vesicles has different reasons. The first reason is a big value of the vesicles polydispersity (48\%) relative to the polydispersity of 500 - $\AA$ extruded vesicles $(27 \%)$. The second reason is the accuracy of the correction for spectrometer resolution. According to Eq. (10), the position of the first minimum in the form factor of infinitely thin sphere depends on the vesicle radius as $q_{R}=\pi / R$, thus $q_{500}=0.0126 \AA^{-1}$ for vesicles with diameter of $500 \AA$ and $q_{1000}=0.0063 \AA^{-1}$ for vesicles with diameter of $1000 \AA$. Figure 5 demonstrates a similar shift of $q_{R}$ for two types of polydispersed vesicle populations of 500- and $1000-\AA$ extruded vesicles. The relative standard deviation of the spectrometer resolution function $\Delta / q=0.53,0.27$, and 0.14 for $q=$ $0.0033,0.0063$, and $0.0126 \AA^{-1}$, respectively (Pedersen et al. 1990). For 1000- $\AA$ extruded vesicles, the accuracy of Eq. (16) decreases in the $q$ region important for the evaluation of the vesicle radius. The value of $q_{1000}=0.0063 \AA^{-1}$ is not so far from the value of $q_{\min }=0.0033 \AA^{-1}$, scattering curve has only five experimental points between these two values. Measurements of the scattering curve with $q_{\min } \ll 0.0033 \AA^{-1}$ and a better spectrometer resolution are necessary for the correct evaluation of the average vesicle radius $\geqslant 500 \AA$. The third possible reason of the underestimation of the vesicle radius for $1000-\AA$ extruded vesicles can be a difference in the statistics of vesicle distribution for 500- and 1000- $\AA$ extruded vesicles. Nonsymmetrical Schulz distribution describes well the 500-A extruded vesicle population. Weibull or some other nonsymmetrical distribution could be more suitable for the characterization of the vesicle population prepared via extrusion thorough 1000-Å pores (Korgel et al. 1998). In any case, careful study of vesicle form and distribution requires the more powerful SANS instrument as discussed above for 1000- $\AA$ extruded vesicles.

Decreasing of the bilayer thickness with decrease of the membrane curvature decreases the number of water molecules $N_{W}$ from $11.9 \pm 0.3$ to $10.8 \pm 0.3$ and increases the surface area $A$ from $61.0 \pm 0.4$ to $62.6 \pm 1.0 \AA^{2}$, whereas the hydrophobic thickness $D$ is unchanged under alteration of the membrane curvature. The structure and hydration of curved DMPC bilayer in the liquid phase depend on the membrane curvature. The bilayer thickness and hydration are altered with increasing of the vesicle radius in the direction of the values for the flat bilayer.

It is known that unilamellar DMPC vesicles are a nonstable system in time (Kiselev et al. 2003b). The membrane thickness and the hydration undergo alteration during system equilibration from the nonstable unilamellar vesicle population to the static state of the multilamellar vesicle population. Probably, the hydration of the DMPC bilayer is a driving force for the transformation of the unilamellar vesicles to the multilamellar one.

DMPC Vesicles in the Liquid Crystalline Phase, the SF Approximation of $\rho(x)$. The SF approximation of scattering length density across the lipid bilayer 
$\rho(x)$ is shown in Fig. 3. The thickness of polar head group $d_{\mathrm{PH}}=9 \AA$ was fixed as in the calculations done earlier (Kucerka et al. 2004). Vesicle shape is considered to be spherical. The difference relative to calculations in (Kucerka et al. 2004) is a free value of the scattering length density in the region of polar head group, $\rho_{\mathrm{PH}}$. The fitting parameters of the lipid bilayer are $d$ and $\rho_{\mathrm{PH}}$. This allows one to make calculation of the water molecules located in the polar head group region $N_{W, \mathrm{PH}}$ and the surface area $A$, taking into account that for the SF approximation the average scattering length density of hydrated DMPC is defined as

$$
\bar{\rho}=\frac{d-2 D_{\mathrm{PH}}}{d} \cdot \rho_{\mathrm{CH}}+\frac{2 D_{\mathrm{PH}}}{d} \cdot \rho_{\mathrm{PH}} .
$$

Table 3. Results for DMPC vesicles in liquid phase $\left(T=30^{\circ} \mathrm{C}\right)$ based on the SF approximation of $\rho(x) . D_{F}$ is diameter of the pores used at extrusion, $\langle R\rangle$ is average vesicle radius, $\sigma$ is vesicle polydispersity, $d$ is membrane thickness, $\rho_{\mathrm{PH}}$ is scattering length density in the region of polar head group, $N_{W, P H}$ and $A$ are number of water molecules in region of polar head group and surface area per DMPC molecule, IB is value of incoherent background. The SFF model

\begin{tabular}{|c|c|c|c|c|c|c|c|}
\hline$D_{F}, \AA$ & $\langle R\rangle, \AA$ & $\sigma, \%$ & $d, \AA$ & $\rho_{\mathrm{PH}}, 10^{10} \mathrm{~cm}^{-2}$ & $N_{W, \mathrm{PH}}$ & $A, \AA^{2}$ & $\mathrm{IB}, \mathrm{cm}^{-1}$ \\
\hline 500 & $275.1 \pm 0.5$ & 27 & $45.5 \pm 0.7$ & $3.7 \pm 0.2$ & $6.8 \pm 0.6$ & $57 \pm 1$ & 0.007 \\
1000 & $296 \pm 2^{*}$ & 48 & $45.7 \pm 0.7$ & $4.0 \pm 0.2$ & $8.0 \pm 0.6$ & $59 \pm 1$ & 0.007 \\
\hline
\end{tabular}

The calculated parameters are given in Table 3. Inside the SF approximation, the membrane thickness $d=45.5 \pm 0.7 \AA$ does not show dependence on the membrane curvature and is the same to the membrane thickness calculated based on the HH approximation for $1000-\AA$ extruded vesicles. Both obtained values of the membrane thickness are larger than the membrane thickness of the giant multilamellar vesicles $(44.2 \AA)$ ). Other fitted and calculated parameters for 500and $1000-\AA$ extruded vesicles are the same within the experimental errors. Two calculated values of $N_{W, \mathrm{PH}}=6.8 \pm 0.6$ and $N_{W, \mathrm{PH}}=8.0 \pm 0.6$ agree well with data for giant multilamellar vesicles 7.2 (Nagle and Tristram-Nagle 2000). Finally, the average value $N_{W, \mathrm{PH}}=7.4 \pm 0.6$ can characterize the number of water molecules in the polar head group of DMPC at $30^{\circ} \mathrm{C}$. Comparison of the hydration calculated by the $\mathrm{HH}$ and SF approximations allows one to calculate the number of water molecules in the region of hydrocarbon chains as $4.5 \pm 0.9$ for 500-A extruded vesicles and $3.4 \pm 0.9$ for 1000 - $\AA$ extruded vesicles. Probably, the decrease of membrane curvature decreases the hydration of the hydrocarbon chains. More precise experiments and data are necessary to answer this question. Surface area $A$ calculated based on the SF approximation has average value $58 \pm 1 \AA$, which a little bit smaller than the value of $59.6 \AA$ for giant multilamellar vesicles (Nagle and Tristram-Nagle 2000). 
DMPC Vesicles in the Gel and Ripple Phases, the SF Approximation of $\rho(x)$. Figure 6 shows the SANS spectrum from $500-\AA$ extruded vesicles measured at $T=10^{\circ} \mathrm{C}$ (gel phase of DMPC) and $T=30^{\circ} \mathrm{C}$ (liquid phase of DMPC). Without consideration of the vesicle polydispersity and the instrument resolution, the first minimum of $F_{s}(q, d)$ corresponds to $q_{R}=\pi / R$. The vesicle radius $R=275.6 \AA$ corresponds to $q_{R}=0.011 \AA^{-1}$. Shift of the $q_{R}$ to the larger value of $q$ reflects the decrease of average vesicle radius with decreasing temperature as seen from Fig. 6 .

The consideration of SANS curves in the Guinier region of $q$ from 0.04 to $0.15 \AA^{-1}$ shows the different exponents at $T=10^{\circ} \mathrm{C}$ and $T=30^{\circ} \mathrm{C}$ (Eq. 3). Visually seen that radius of gyration of the bilayer $R_{t}$ increases with decreasing temperature from 30 to $10^{\circ} \mathrm{C}$. This simple analysis corresponds to the dependence of the membrane thickness parameter $d_{G}$ on temperature as shown in Figs. 1 and 7.

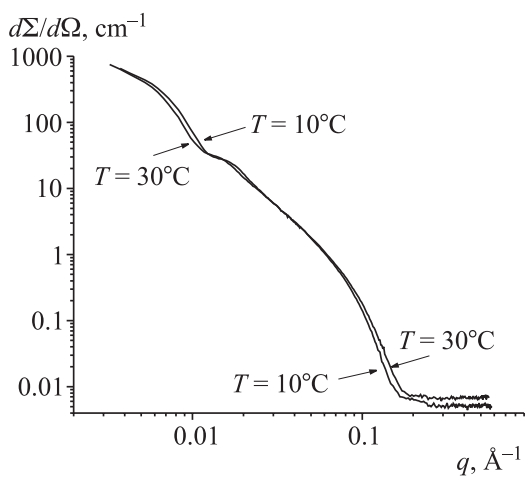

Fig. 6. Experimental macroscopic cross sections of the unilamellar vesicle populations at $T=30^{\circ} \mathrm{C}$ and $T=10^{\circ} \mathrm{C}$ for vesicles prepared by extrusion through pores of $500-\AA$ diameter

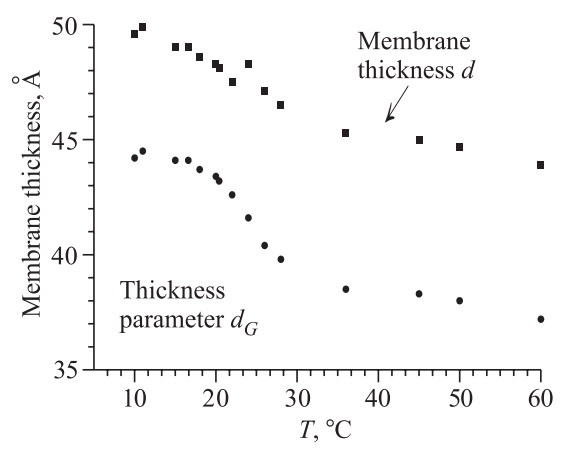

Fig. 7. Dependence of DMPC membrane thickness parameter $d_{G}$ and membrane thickness $d$ on temperature for $1000-\AA$ extruded vesicles

The number of water molecules in the bilayer depends on lipid phase. For giant multilamellar dipalmitoylphosphatidylcholine (DPPC) vesicles, the number of water molecules in the region of polar head groups decreases from 8.6 in liquid phase to 3.7 in gel phase (Nagle and Tristram-Nagle 2000). The SF approximation of $\rho(x)$ with fixed thickness of the polar head region $d_{\mathrm{PH}}$ is more appropriate for gel and ripple phases of the DMPC bilayer (Fig.3). Value of $\rho_{\mathrm{CH}}=-0.39 \cdot 10^{10} \mathrm{~cm}^{-2}$ was used as fixed parameter in the calculations for gel and ripple phases of DMPC.

Measured SANS curves at $T=10$ and $20^{\circ} \mathrm{C}$ for 500 - and 1000 - $\AA$ extruded vesicles were fitted based on the SF approximation of $\rho(x)$. The deformation of spherical shape to elliptical was taken into account. Opposite the liquid phase, 
Table 4. Results for DMPC 500- $\AA$ extruded vesicles in gel $\left(T=10^{\circ} \mathrm{C}\right)$ and ripple $\left(T=20^{\circ} \mathrm{C}\right.$ ) phases based on the SF approximation of $\rho(x) . T$ is temperature, $\langle a\rangle$ is average value of minor semiaxis, $\varepsilon$ is eccentricity, $\sigma$ is vesicle polydispersity, $d$ is membrane thickness, $\rho_{\mathrm{PH}}$ is scattering length density of polar head group, $N_{W, \mathrm{PH}}$ and $A$ are number of water molecules in region of polar head group and surface area per DMPC molecule, IB is value of incoherent background. The SFF model

\begin{tabular}{|c|c|c|c|c|c|c|c|c|}
\hline$T,{ }^{\circ} \mathrm{C}$ & $\langle a\rangle, \AA$ & $\varepsilon$ & $\sigma, \%$ & $d, \AA$ & $\rho_{\mathrm{PH}}, 10^{10} \mathrm{~cm}^{-2}$ & $N_{W, P H}$ & $A, \AA^{2}$ & $\mathrm{IB}, \mathrm{cm}^{-1}$ \\
\hline 10 & $185 \pm 1$ & 1.62 & 21 & $49.1 \pm 0.7$ & $3.7 \pm 0.2$ & $5.2 \pm 0.5$ & $48.8 \pm 0.9$ & 0.005 \\
20 & $187 \pm 1$ & 1.63 & 22 & $47.9 \pm 0.7$ & $3.6 \pm 0.3$ & $5.3 \pm 0.5$ & $50 \pm 1$ & 0.006 \\
\hline
\end{tabular}

Table 5. Results for DMPC 1000- $\AA$ extruded vesicles in gel $\left(T=10^{\circ} \mathrm{C}\right)$ and ripple $\left(T=20^{\circ} \mathrm{C}\right.$ ) phases based on the SF approximation of $\rho(x)$. $T$ is temperature, $\langle R\rangle$ is average vesicle radius, $\sigma$ is vesicle polydispersity, $d$ is membrane thickness, $\rho_{\mathrm{PH}}$ is scattering length density of polar head group, $N_{W, \mathrm{PH}}$ and $A$ are number of water molecules in region of polar head group and surface area per DMPC molecule, IB is value of incoherent background. The SFF model

\begin{tabular}{|c|c|c|c|c|c|c|c|}
\hline$T,{ }^{\circ} \mathrm{C}$ & $\langle R\rangle, \AA$ & $\sigma, \%$ & $d, \AA$ & $\rho \mathrm{PH}, 10^{10} \mathrm{~cm}^{-2}$ & $N_{W, \mathrm{PH}}$ & $A, \AA^{2}$ & $\mathrm{IB}, \mathrm{cm}^{-1}$ \\
\hline 10 & $309.0 \pm 0.4^{*}$ & 37 & $49.6 \pm 0.5$ & $3.6 \pm 0.2$ & $5.9 \pm 0.5$ & $49.2 \pm 0.9$ & 0.006 \\
20 & $316 \pm 1^{*}$ & 35 & $48.3 \pm 0.6$ & $3.8 \pm 0.2$ & $5.9 \pm 0.4$ & $50.4 \pm 0.8$ & 0.006 \\
\hline
\end{tabular}

extruded $500-\AA$ vesicles in gel and ripple phases have pronounced elliptical form with eccentricity $\varepsilon=1.6$. Extruded $1000-\AA$ vesicles have $\varepsilon=1.1$ and can be considered as spherical taking into account the problems in the determination of vesicle radius. The internal membrane structure did not show the dependence on the eccentricity and membrane curvature for both vesicle populations. The results of fitting and calculation are given in Table 4 for extruded 500-A vesicles in gel $\left(T=10^{\circ} \mathrm{C}\right)$ and ripple $\left(T=20^{\circ} \mathrm{C}\right)$ phases. Results for extruded $1000-\AA$ vesicles are given in Table 5. The average values of the minor semiaxis $a=185$ and the major semiaxis $b=300$ at $T=10^{\circ} \mathrm{C}$ change to $a=187$ and $b=303$ at $T=20^{\circ} \mathrm{C}$ for vesicles prepared by extrusion through pores with a $500-\AA$ diameter. These values are in agreement with radius of pores. Similar to the liquid DMPC phase at $30^{\circ} \mathrm{C}$, the average radius of $1000-\AA$ extruded vesicles is underestimated relative to the radius of pores and DLS results. Polydispersity of vesicle population has smaller values in gel and ripple phases relative to the liquid phase. For 500- $\AA$ extruded vesicles, the polydispersity increases from the value of $21 \%$ in gel phase to the value of $26 \%$ in liquid phase. The average area of the $500-\AA$ extruded vesicles $S=4 \pi \varepsilon a^{2}$ is equal to $9.9 \cdot 10^{5} \AA^{2}$ at $T=30^{\circ} \mathrm{C}$. Average area of the vesicle decreases to the values of $7.2 \cdot 10^{5} \AA^{2}$ in ripple and $7.0 \cdot 10^{5} \AA^{2}$ in gel phases. The decrease of vesicle area during the phase transitions from liquid to 
gel phase should increase the membrane thickness. Membrane thickness increases from $47.8 \pm 0.2 \AA$ at $30^{\circ} \mathrm{C}$ to $49.1 \pm 0.7 \AA$ at $10^{\circ} \mathrm{C}$.

Unlike the liquid DMPC phase, the difference in the membrane curvature for 500- and 1000- $\AA$ extruded vesicles has no influence on the membrane thickness and hydration in gel and ripple phases. Values of the membrane thickness $d=$ $49.6 \pm 0.5 \AA$ and the surface area $A=49.2 \pm 0.9 \AA^{2}$ for $1000-\AA$ extruded vesicles are a little bit larger than the values $d=48.2 \AA$ and $A=47 \AA^{2}$ determined for giant multilamellar DMPC vesicles in gel phase (Tristram-Nagle et al. 2002). Giant multilamellar DMPC vesicles can be considered as a membrane with zero curvature. For gel phase, numbers of water molecules in the region of polar head groups per DMPC molecule $N_{W, \mathrm{PH}}$ obtained for 500- and 1000- $\AA$ extruded vesicles are the same. Average value of $N_{W, \mathrm{PH}}=5.6 \pm 0.5$ obtained in gel phase is smaller than the hydration of liquid phase, $N_{W, \mathrm{PH}}=7.4 \pm 0.6$. This difference in the hydration of gel and liquid phases is sufficiently smaller than the corresponding difference in the hydration of DPPC.

Opposite to liquid and gel phases of DMPC, information about internal membrane structure and hydration in ripple phase is hard to be evaluated from the $\mathrm{X}$-ray diffraction experiment due to the limited number of the diffraction peaks. Parameters of the vesicle population and the bilayer structure of DMPC vesicles in ripple phase are given in Tables 4 and 5. For 500- $\AA$ extruded vesicles, the evaluated vesicle parameters are the same in gel and ripple phases within the range of experimental errors. For $1000-\AA$ extruded vesicles, the membrane thickness decreases from the value of $49.6 \pm 0.5 \AA$ in gel phase to $48.3 \pm 0.6 \AA$ in ripple phase. Finally, one can use parameters evaluated for $1000-\AA$ extruded vesicles $d=48.3 \pm 0.6 \AA, N_{W, \mathrm{PH}}=5.9 \pm 0.4$, and $A=50.4 \pm 0.8 \AA$ to characterize DMPC bilayer structure in ripple phase.

Guinier Approximation for the Flat Membrane, Connection with the SFF Model. It is commonly believed that Guinier approximation describes the SANS curve at $q \rightarrow 0$. It is true for globular particles with one typical size (Guinier and Fournet 1955). Vesicles have two typical sizes: radius and membrane thickness, which are different in the scale of one-two orders of a magnitude. SANS curve from vesicles have two Guinier regions. First one is Guinier region of the vesicle size at $q \rightarrow 0$ and other one is Guinier region of the membrane. The sense and validity of the membrane Guinier approximation is clarified below.

Let one consider the lipid membrane as membrane with $\rho_{c}(x) \equiv \Delta \rho=$ const. This approximation conventionally named as homogeneous approximation (Feigin and Svergun 1987). Really, lipid membrane is a nonhomogeneous object, $\rho_{c}(x) \neq$ const. Homogeneous approximation can describe only some part of the experimental scattering curve (Feigin and Svergun 1987). This part of the curve can be obtained via a comparison of experimental and calculated SANS curves (Kiselev et al. 2002). According to the SFF model, the scattering amplitude of 
vesicle for $\rho_{c}(x) \equiv \Delta \rho=$ const and $R \gg d_{G}$ is written as (Kiselev et al. 2002)

$$
A(q)=\left(4 \pi R^{2} \cdot \frac{\sin (q R)}{q R}\right) \cdot\left(\frac{2 \Delta \rho}{q} \cdot \sin \left(\frac{q d_{G}}{2}\right)\right) .
$$

The approximation of the harmonic function by exponent

$$
\frac{\sin x}{x} \approx e^{-(x / \sqrt{6})^{2}}
$$

is valid for $x<1$. Equation (22) can be rewritten as

$$
A(q)=\left(S \cdot \frac{\sin (q R)}{q R}\right) \cdot \Delta \rho \cdot d_{G} \cdot \exp \left(-\frac{\left(q d_{G}\right)^{2}}{24}\right),
$$

where $S=4 \pi R^{2}$ is the vesicle surface area. It is important to note that transition from the harmonic function to the exponent has no request of $q \rightarrow 0$. The validity of this transformation is $q \cdot d_{G} / 2<1$. The exponential presentation of the scattering amplitude is the principal property of the Guinier approximation. For $q<2 / d_{G}$, the vesicle form factor $F=A^{2}$ is written as

$$
F(q)=\left(\frac{4 \pi S \cdot \sin ^{2}(q R)}{q^{2}}\right) \cdot(\Delta \rho \cdot d)^{2} \cdot \exp \left(-\frac{\left(q d_{G}\right)^{2}}{12}\right) .
$$

Let us consider the flat lipid bilayer, which corresponds to $R \rightarrow \propto$. In this case, $\Delta q=\pi / R \rightarrow 0$, where $\Delta q$ is distance between minima of the $\sin ^{2}(q R)$. Any SANS spectrometer has uncertainty in the values of measured $q$ and as well as any vesicle population has uncertainty in the value of $R$. One can therefore use average value $\overline{\sin ^{2}(q R)}=1 / 2$ instead of $\sin ^{2}(q R)$. Using Eq. (4) for $R_{t}$, finally one obtains

$$
F(q)=\left(\frac{2 \pi S}{q^{2}}\right) \cdot\left(\Delta \rho \cdot d_{G}\right)^{2} \cdot \exp \left(-q^{2} \cdot R_{t}^{2}\right) .
$$

Equation (26) is Guinier approximation of the form factor for the flat bilayer with infinitely large area $S$ and membrane radius of gyration $R_{t}$. Form factor of the infinitely thin flat membrane has $1 / q^{2}$ behavior. Form factor of the membrane with thickness parameter $d_{G}$ has exponential form and characterizes at $q \rightarrow 0$ by asymptotic properties of the function $F(q) \cdot q^{2}$.

Equation (26) was evaluated from SFF model on the basis of two approximations: $\Delta \rho=$ const and $R \rightarrow \propto$. This equation limits a possibility to receive some information about internal membrane structure. Internal membrane structure is a case of $\Delta \rho \neq$ const and requires $q$ values around the region of Guinier. It 
can explain the difference between the values of DMPC membrane thickness for 500- $\AA$ A extruded vesicles at $T=30^{\circ} \mathrm{C}$ obtained via application of the Guinier approximation, $d=44.5 \pm 0.3 \AA$ and presented in Tables $3 d=45.5 \pm 0.7 \AA$ (Kucerka et al. 2004).

Dependence of the membrane thickness parameter $d_{G}$ on temperature as shown in Fig. 1 can be corrected now for the values of membrane thickness calculated for $1000-\AA$ extruded vesicles at 10,20 , and $30^{\circ} \mathrm{C}$. The obtained values of parameter $\Delta d_{H}$ are 5.4, 4.9, and $6.7 \AA$ respectively for 10,20 , and $30^{\circ} \mathrm{C}$. Membrane thickness $d$ is calculated using Eq. (5) on the assumption that $\Delta d_{H}$ has permanent values in gel, ripple, and liquid phases. Figure 7 shows the obtained dependence of the DMPC membrane thickness on temperature for 1000$\AA$ extruded vesicles. The main feature in temperature dependence $d(T)$ is a sharp increase in the membrane thickness at the temperature of the main phase transition, $T=23^{\circ} \mathrm{C}$. Obtained anomalous $d(T)$ behavior supports the critical fluctuations of membrane thickness rather than fluctuations of water layer at the temperature of main phase transitions (Zhang et al. 1995, Lemmich et al. 1995).

\section{CONCLUSIONS}

The Separated Form Factor model of the small-angle scattering from vesicles can be used for data interpretation for vesicles with radius larger than $250 \AA$. Shultz distribution describes well the vesicle shape and size for vesicles prepared via extrusion through pores of $500 \AA$ A. Average vesicle shape is near spherical (eccentricity 1.1) for liquid phase of DMPC and deviates sufficiently from sphere in gel and ripple phases (eccentricity 1.6). The average vesicle radius and the polydispersity can be evaluated correctly from SANS curve with minimum measured scattering vector $q_{\min }=0.0033 \AA^{-1}$ for vesicles with radius of about $250 \AA$ A. It should be emphasized that SANS experiment with $q_{\min }<0.0033 \AA^{-1}$ is necessary for correct evaluation of radius $\geqslant 500 \AA$. Approximation of the scattering length density across DMPC bilayer in liquid phase as hydrophobic and hydrophilic regions with linear water distribution describes the internal bilayer structure without any preliminary structural information. The Step Function approximation of the scattering length density allows an evaluation of DMPC membrane thickness and scattering length density of the polar head group in gel, ripple and liquid phases based on the preliminary structural information about thickness of the polar head group. It was shown that thickness of the bilayer depends on the membrane curvature. The DMPC bilayer thickness of the curved unilamellar vesicles $(49.6 \pm 0.5$ and $45.5 \pm 0.6 \AA$ in gel and liquid phases, respectively) is larger relative to that of multilamellar vesicles with nearly flat membrane (48.2 and $44.2 \AA$ in gel and liquid phases, respectively). Interpretation of the SANS data is based on the calculation of the scattering length density across the membrane bilayer. Information about specific volume of the lipid molecule 
allows one to receive additional structural information from SANS. In our study, a preliminary knowledge of the DMPC molecular volume allows one to calculate bilayer hydration and lipid surface area. The X-ray diffraction has been successfully applied to characterize the internal membrane structure in gel and liquid phases. However, the diffraction normally does not give accurate information about membrane structure in ripple phase. This drawback of diffraction technique can be overcome by SANS. The internal membrane structure and hydration of ripple DMPC phase were evaluated from the SANS experiment in present article.

The results presented here emphasize the importance of appropriate models and approximations for the interpretation of SANS data. Usefulness of SANS technique for the characterization of the internal bilayer structure and hydration was shown for vesicular systems. Thus herein presented methods can be used for the characterization of vesicular-based drug delivery systems.

Acknowledgements. This work is partly based on the experiments performed at Swiss spallation neutron source SINQ, Paul Scherrer Institute, Villigen, Switzerland. The authors are grateful to Prof. Dr. V.L. Aksenov for support of this study, Prof. Dr. H. Schmiedel and Prof. Dr. A. M. Balagurov for fruitful discussions. The investigation was supported by the Ministry of Science and Technology RF (contract No. 40.012.1.1.1148), Grant of Leading Scientific Scholl, and RFBR (grant No.03-01-00657). The authors would like to thank Lipoid (Moscow) for the gift of DMPC.

\section{REFERENCES}

1. Armen P. S., Uitto O.D., Feller S. E. (1998) Phospholipid component volumes: determination and application to bilayer structure calculations // Biophys. J. 75: 734-744.

2. Balgavy P. et al. (1998) Bilayer thickness in unilamellar extruded egg yolk phosphatidylcholine liposomes: a small-angle neutron scattering study // Acta Physica Slovaca 48: 509-533.

3. Balgavy P. et al. (2001) Bilayer thickness and lipid interface area in unilamellar extruded 1,2-diacylphosphatidylcholine liposomes: A small-angle neutron scattering study // Biochim. Biophys. Acta 1521: 40-52.

4. Cevc G., Schatzlein A., Richardsen H. (2002) Ultradiformable lipid vesicles can penetrate the skin and other semi-permiable barriers unfragmented. Evidence from double label SLSM experiments and direct size measurements // Biochim. Biophys. Acta 1564: $21-30$.

5. Feigin L.A., Svergun D. I. (1987) Structure analysis by small-angle X-ray and neutron scattering. N. Y.: Plenum Publishing Corporation.

6. Glatter O. (1977) Data evaluation in small-angle scattering: calculation of the radial electron density distribution by means of indirect Fourier transformation // Acta Physica Austriaca 47: 83-102. 
7. Glatter O. (1980) Evaluation of small-angle scattering data from lamellar and cylindrical particles by the indirect transformation method // J. Appl. Cryst. 13: 577-584.

8. Gordeliy V. I. et al. (1993) The study of single biological and model membranes via small-angle neutron scattering // Progr. Colloid. Polym. Sci. 92: 252-257.

9. Gordeliy V.I., Kiselev M. A. (1995) Definition of lipid membrane structural parameters from neutronographic experiments with the help of the strip function model // Biophys. J. 69: 1424-1428.

10. Guinier A., Fournet G. (1955) Small-angle scattering of X-rays. N. Y.: John Wiley \& Sons, Inc.

11. Gutberlet T. et al. (2000) SANS study of mixed POPC/C ${ }_{12} E_{n}$ aggregates // Physica B 381-383: 276-278.

12. Hallet F.R., Watton J., Krygsman P. (1991) Vesicle sizing. Number distributions by dynamic light scattering // Biophys. J. 59: 357-362.

13. Jin A. J. et al. (1999) Light scattering characterization of extruded lipid vesicles // Eur. Biophys. J. 28: 187-199.

14. Kiselev M. A. et al. (2001). Sucrose solutions as prospective medium to study the vesicle structure: SAXS and SANS study // J. Alloys Compounds 328: 71-76.

15. Kiselev M.A. et al. (2002) Model of separated form factors for unilamellar vesicles // J. Appl. Phys. A 74: S1654-S1656.

16. Kiselev M. A. et al. (2003a) Structure factor of dimyristoylphosphatidylcholine unilamellar vesicles: small-angle X-ray scattering study // Surface 11: 20-24 (in Russian).

17. Kiselev M. A. et al. (2003b). Does sucrose influence the properties of DMPC vesicles? // Chem. Phys. Lipids 123: 31-44.

18. Kiselev M. A., Zemlyanaya E. V., Aswal V. K. (2004) SANS study of unilamellar DMPC vesicles: Fluctuation model of a lipid bilayer // Crystallography Reports 49: s136s141.

19. Kiselev M.A. et al. (2005) New insights into structure and hydration of stratum corneum lipid model membrane by neutron diffraction // Europ. Biophys. (in press).

20. Knoll W. et al. (1981) Small-angle neutron scattering of aqueous dispersions of lipids and lipid mixtures. A contrast variation study // J. Appl. Cryst. 14: 191-202.

21. Korgel B., van Zanten J.H., Monbouquette H. G. (1998) Vesicle size distributions measured by flow field-flow fractionation // Biophys. J. 74: 3264-3272.

22. Kucerka N., Kiselev M. A., Balgavy P. (2004) Determination of bilayer thickness and lipid surface area in unilamellar dimyristoylphosphatidylcholine vesicles from smallangle neutron scattering curves: a comparison of evaluation methods // Eur. Biophys. J. 33: $328-334$.

23. Lemmich J. et al. (1995) Pseudocritical behaviour and unbinding of phospholipids bilayers // Phys. Rev. Lett. 75: 3958-3961.

24. MacDonald R. C. et al. (1991) Small-volume extrusion apparatus for preparation of large, unilamellar vesicles // Biochim. Biophys. Acta 1061: 297-303.

25. Nagayasu A., Uchiyama K., Kiwada H. (1999) The size of liposomes: a factor which affects their targeting efficiency to tumors and therapeutics activity of liposomal antitumor drugs // Adv. Drug Delivery Rev. 40: 75-87. 
26. Nagle J. F., Tristram-Nagle S. (2000) Structure of lipid bilayers // Biochim. Biophys. Acta 1469: 159-195.

27. Ostanevich Yи.M. (1988) Time-of-flight small-angle scattering spectrometers on pulsed neutron sources // Macromol. Chem. Macromol. Symp. 15: 91-103.

28. Patty P.J., Frisken B.J. (2003) The pressure-dependence of the size of extruded vesicles // Biophys. J. 85: 996-1004.

29. Pedersen J.S. (1997) Studies of soft condenced matter by SANS, SAXS and reflectometry // 5th Summer School on Neutron Scattering, Lyceum Alpinum, Zuoz, Switzerland.

30. Pedersen J. S., Posselt D., Mortensen K. (1990) Analytical treatment of the resolution function for small-angle scattering // J. Appl. Cryst. 23: 321-333.

31. Pencer J., Hallet R. (2000): Small-angle neutron scattering from large unilamellar vesicles: An improved method for membrane thickness determination // Phys. Rev. E 61: 3003-3008.

32. Pencer J., White G.F., Hallet F.R. (2001) Osmotically induced shape changes of large unilamellar vesicles measured by dynamic light scattering // Biophys. J. 81: 2716-2728.

33. Schmiedel H. et al. (2001) Determination of structural parameters and hydration of unilamellar POPC/C12E4 vesicles at high water excess from neutron scattering curves using a novel method of evaluation // J. Phys. Chem. B 105: 111-117.

34. Schmiedel H. et al. (2004) Multilamellarity, structure and hydration of extruded POPC vesicles by SANS. Condensed Matter Physics with Neutrons at the IBR-2 Pulsed Reactor. JINR, E14-2004-148. Dubna, 2004.

35. Silin I. N. (1967) Standard minimization code on the base of the least square method. JINR Commun. 11-3362. Dubna, 1967.

36. Tristram-Nagle S. et al. (2002) Structure of gel phase DMPC determined by X-ray diffraction // Biophys. J. 83: 3324-3335.

37. Wiener M. C., White S. H. (1991) Fluid bilayer structure determination by the combined use of X-ray and neutron diffraction // Biophys. J. 59: 162-173.

38. Zhang R. et al (1995) Critical fluctuations in membranes // Phys. Rev. Lett. 74: 2832-2835.

39. Zemlyanaya E. V., Kiselev M.A. (2002) Determination of the unilamellar dimyristoylphosphatidylcholine vesicle structure from the small-angle scattering data. JINR Commun. P3-2002-163. Dubna, 2002.

40. Zemlyanaya E. V., Kiselev M.A., Aswal V.K. (2005) Structure of the unilamellar dimyristoylphosphatidylcholane vesicles. A small-angle neutron scattering study // J. Comput. Meth. Appl. Sci. Engineer. (in press); physics/0411029.

Received on June 27, 2005. 
Редактор Н. С. Скокова

Подписано в печать 08.09.2005.

Формат $60 \times 90 / 16$. Бумага офсетная. Печать офсетная.

Усл. печ. л. 1,44. Уч.-изд. л. 2,1. Тираж 280 экз. Заказ № 55002.

Издательский отдел Объединенного института ядерных исследований 141980, г. Дубна, Московская обл., ул. Жолио-Кюри, 6.

E-mail: publish@pds.jinr.ru www.jinr.ru/publish/ 